\title{
Vibration Behavior and Rebound Angle on the Collision of Mirror Models inside a SLR Camera*
}

\author{
Hiroki MATSUMOTO ** and Masaki HIRASHIMA** \\ **Muroran Institute of Technology \\ 27-1 Muzumono-cho, Muroran-shi, Hokkaido, Japan \\ E-mail:h_matsu@mmm.muroran-it.ac.jp
}

\begin{abstract}
In this study, the rebound vibration characteristics of the internal mirror of SLR camera were investigated using experimental models. The mechanism of the mirror rebound phenomena is tested by using six types of rectangular metal plate models. The mirror (plates) model is supported by fixing its longitudinal edge on a horizontal rotatable shaft. The mirror plate swings down freely around a horizontal axis and hits with a stopper and this phenomenon simulate the motion. A laser displacement meter is used to measures the amount of rebound. The result reveals that the rebound amount and the vibration characteristics after the collision depend on the stopper position. Also, the vibration after the collision depends on the collision behavior and when the amount of rebound is small, there is a tendency that the vibration after the collision becomes large.
\end{abstract}

Key words: Vibration of Mechanism, Vibration of Continuous System, Kinematic, Nonlinear Vibration

\section{Introduction}

Single-Lens reflex cameras (SLR Camera) permit a photographer to see a photogenic object through the optical viewfinder. For this, SLR cameras have a moving mirror system in it. Before the shutter-release button is pressed, the mirror is positioned between the lens and the shutter curtain. The ray from the photogenic object through the lens reach the internal mirror consists of a half mirror and a sub mirror. The mirror system guides a part of the ray upwards to the viewfinder. At the same time, rest of the ray is filtered out to the downward direction into the auto focus sensor. At this instance, the photographer can confirm the focus of the object in the viewfinder. After focusing the camera, the photographer presses the shutter-release button. When the release button is pressed, the mirror swings up to the upper side of the camera and allows the rays that are coming through the lens to proceed straight through to the shutter curtain. And then, the shutter curtain opens and an image pickup device or film is exposed. Then, the mirror swings back down and strikes on the mirror stopper. At this moment, the collision force is applied on the mirror and the rebound vibration occurs. While the mirror is in vibration, the autofocus mechanisms cannot perform well. Therefore the camera does not take a next picture continuously. For high-speed and precision autofocusing control, the rebound vibration suppressing mechanism is required. The high performance SLR cameras have rebound reducing mechanism and that leads to high production cost. Low cost SLR cameras do not have the rebound reduction mechanism and with this study we seek the new method for the 
vibration suppression without additional devices for reduction of rebound phenomena. In order to find the method, we think that it is need to clarify the essential mechanisms of the rebound phenomena at first. For that purpose, it is necessary to change the stopper position and mirror structure and associated components.

The previous studies on collision behavior of a tennis racket or a butt and a ball ${ }^{(1)-(6)}$ have considered the eigenvalue and vibration modes to describe the rebound phenomena. Other point of view is mechanical energy of collision phenomena. From the previous study, we can analogize the mirror rebound phenomena related with the vibration behavior of the mirror. However, in the study of the tennis rackets, the rebound object is the ball and the vibrating object is the racket. But in our case the mirror vibrates and rebounds itself. Thus, we need to explain the phenomena for the bouncing object. We could not find similar studies that summarize the behavior of the rigid body and elastic vibration of a plate that rebound post-collision.

Based on those reason, we thought the necessity of understanding the essential mechanism of rebound phenomena. Thus, in this study, we performed fundamental experiments on the rebound angle of simple mirror models of SLR camera. The actual mirror system has roughly three components that are a half mirror, a sub-mirror and a base structure. The actual mirror system includes some problems at the same instant that are an adhesion problem of the half mirror and a dynamic problem of the sub-mirror. In order to simplify the problem, we use a simple model of actual mirror that has same outline of the base structure. In the actual system, the base structure contacts with the stopper. In the experiments, the effects of stopper position and the effects of mirror shape on the amount of rebound angle were examined. And then, in order to understand the rebound phenomenon, the vibration behavior of the mirror at the moment of collision at the stopper is measured. Here, we focus mainly on the relationship between the stopper position and the rebound angle to find out the effects of the stopper position on the rebound angle. Moreover, we consider mechanisms of the rebound phenomenon from the results of measurement of elastic vibration behavior.

\section{Nomenclature}

$x_{s}:$ stopper position along $x$-axis

$y_{s}:$ stopper position along $y$-axis

$V_{d}$ : vertical displacement after the collision with the stopper

$\theta:$ angle of between stopper and mirror model

$\theta_{r}:$ rebound angle of mirror model

\section{Experimental equipment and method}

\subsection{Mirror models}

Fig. 1 shows the mirror models used in this study. The actual mirror has some parts in a mirror system, for example, a sub mirror, support frame etc. The mirror models are made out of aluminum plates with $1 \mathrm{~mm}$ thick rectangular plates with $60 \times 80 \mathrm{~mm}$ in size. The mirror model shown in Fig.1 (a) is the scaled up model and the simplest model. The mirror models are bolted on the shaft by M3 bolts with jig. The mirror model rotates with jigs and shaft. The shaft diameter is $4 \mathrm{~mm}$ and attached to the main body by ball bearings. In order to measure the vibration behavior of mirror model with high resolution scaled up mirror models were used. In order to understand the scaling effect, preliminary experiments were done to measure the rebound angle for scaling up mirror model (a) and actual size mirror model (small model). The size of the small mirror model is $30 \times 40 \mathrm{~mm}$ and $1 \mathrm{~mm}$ thickness. The small model is made of aluminum and it has two shaft holes (Fig.2). In the experiment, we measured the rebound angle by varying the stopper positions in $x$-axis and $y$-axis direction, respectively. As shown in Fig.2, the stopper position that indicates lowest rebound 
angle is about the same at about $x_{s} / L_{x}=0.6-0.7$ for both cases. Where $L_{x}$ is the length of the leading edge of the mirror model. Also we find the similar characteristic of inclination of rebound angle for $y$-direction stopper potion, too. This results show that the size of the model does not have significant effect on the rebound angle. Thus the large mirror model is used in the experiments.

In actual SLR camera, the stopper position is only located at y-direction. Therefore the stopper potion is only located to y-direction. The Mirror model (b) shown in Fig.1 has a rectangle hole with $40 \times 20 \mathrm{~mm}$ size on the center of the model. This simulates the hole provided in an actual mirror in order to guide the ray into auto focus sensor. In order to investigate the effects of a slit on the mirror, models (c) - (f) have provided a slit of width is $4 \mathrm{~mm}$.

Fig.3 shows the location of the stopper reference to the mirror model. The stopper position is determined by distance $y_{s}$ from the model edge as shown and $y_{s}$ can vary from 0 $\mathrm{mm}$ to $42 \mathrm{~mm}$ by $2 \mathrm{~mm}$ increments. The rebound angle is measured every stopper position of $y_{s}$. The stopper position kept same in all mirror models. The stopper is an aluminum column with $5 \mathrm{~mm}$ diameter, and $1 \mathrm{~mm}$ length of the column contacts with the mirror model.

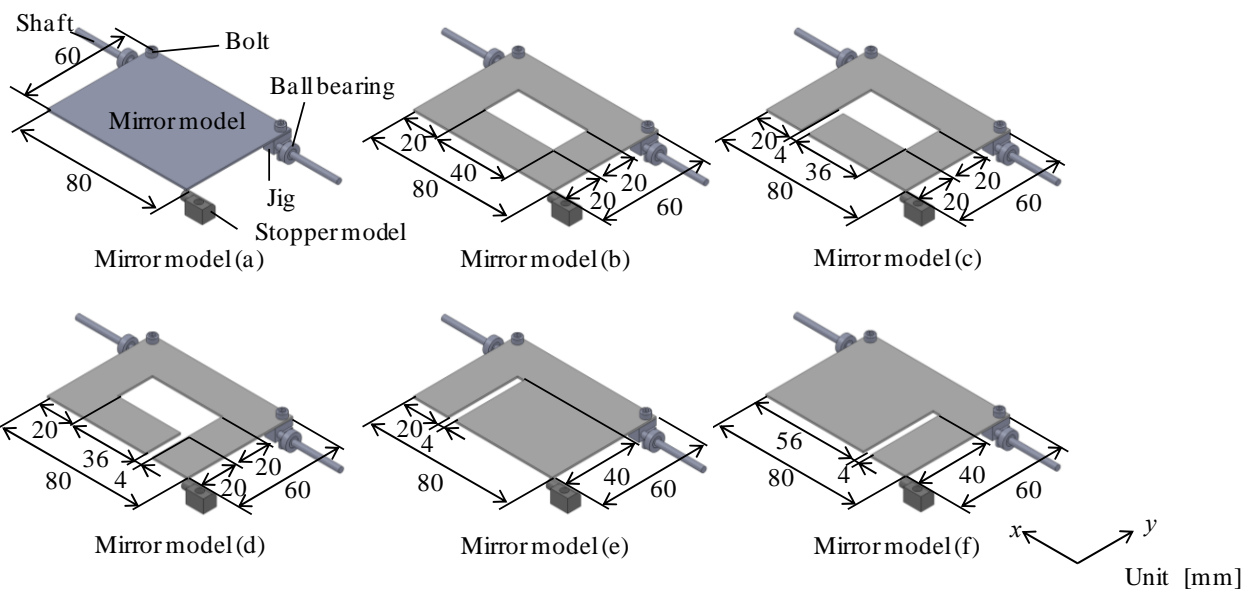

Fig.1 Mirror models

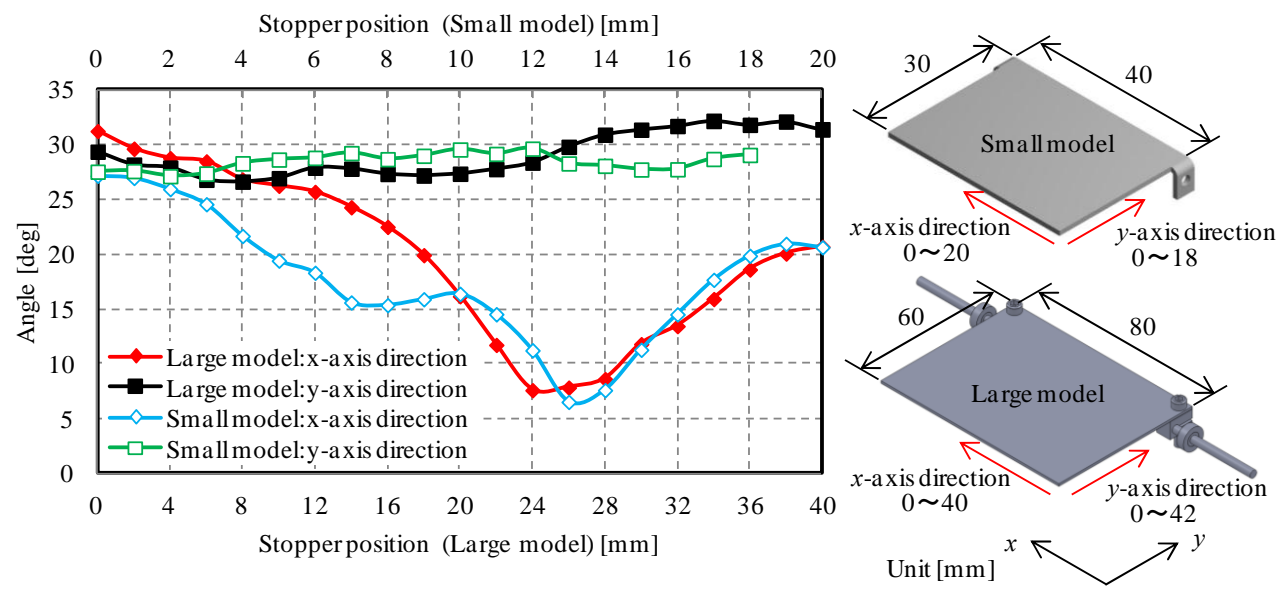

Fig.2 Preliminary experimental result

\subsection{Measurement of rebound angle ${ }^{(7)}$}

In order to find the effect of stopper position on the rebound angle, the amount of rebound angle of mirror models were measured. Fig. 4 shows the experimental setup. The stopper is located at $45 \mathrm{deg}$ downward from the axis of rotation of the shaft. The mirror 
model freely swings down from horizontal plane at hits at the stopper. As shown in Fig.3 the measurement point of rebound angle is located and the laser displacement meter was used to measure the displacement of the mirror model. And then, A/D converter and PC were used to record the time series data. The measured displacement is converted to mirror model's angle $\theta_{r}$. The maximum rebound displacement in the time series data was defined as the rebound angle of the mirror model. The measurements were repeated 5 times per each set and the average was taken as the rebound angle. The data maximum variability is $0.9 \mathrm{deg}$, and the average variability is about $0.4 \mathrm{deg}$.

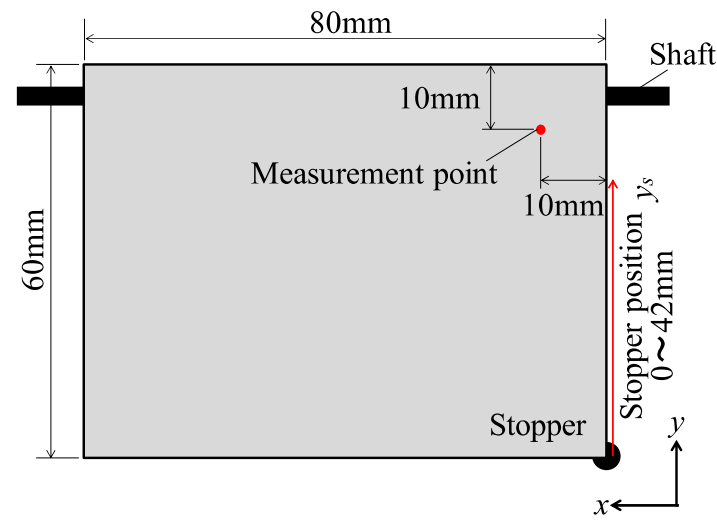

Fig.3 Stopper potions and measurement point

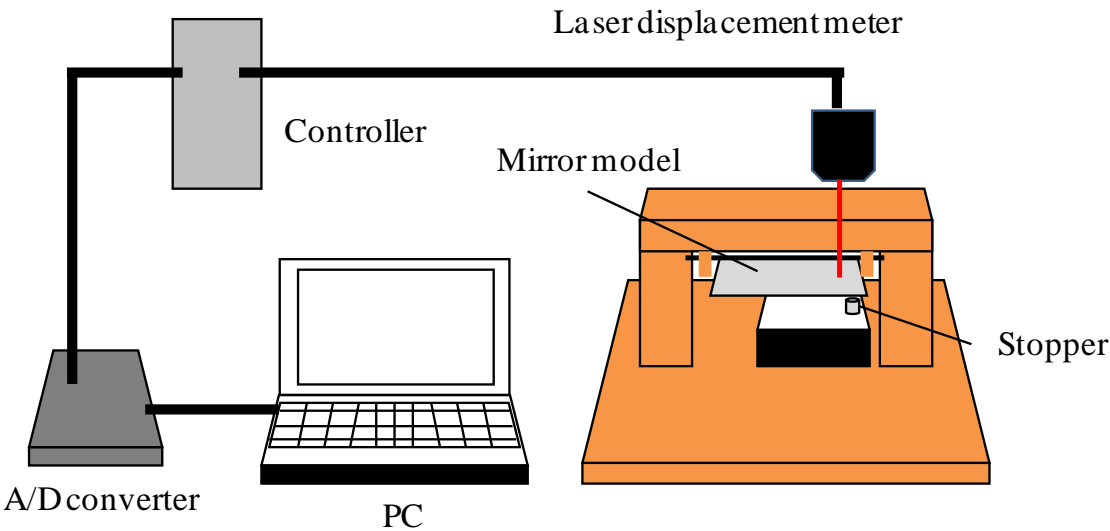

Fig.4 Schematic diagram of experimental equipment

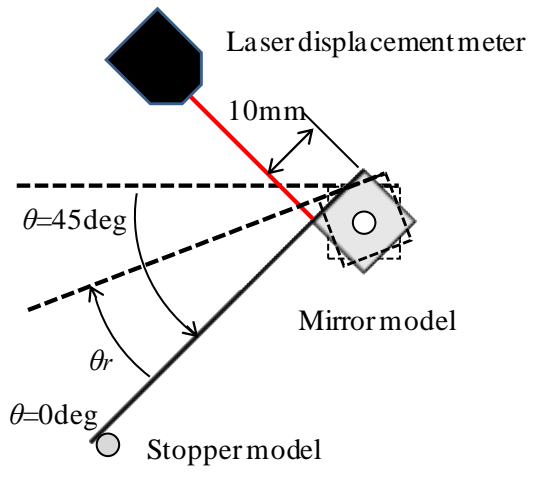

Fig.5 Measurement method of the amount of rebound

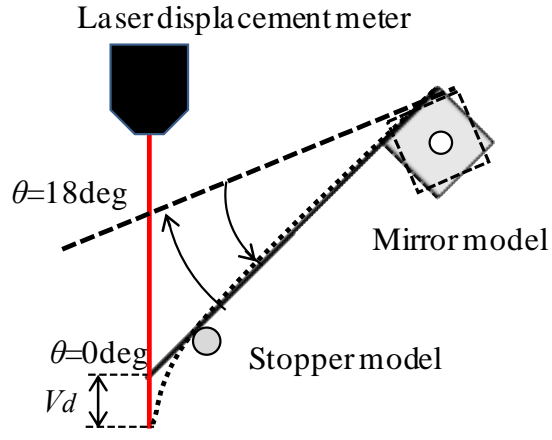

Fig.6 Measurement method of the vibration behavior of mirror models 


\subsection{Calculation of rebound angle}

The laser displacement sensor only can measure vertical displacement of the mirror rebound. The rebound amount is estimated by the rebound angle of the mirror. Therefore, the displacement is converted to the angle using geometrical relationship. As shown in Fig.7, we find $\theta=\angle C B D=\angle B O A, \angle C D B=\angle B A O=90$ deg, therefore $\triangle C B D$ and $\triangle B O A$ are a similar triangle. From $\triangle O A B$ and $\mathrm{OA}=\mathrm{a}$, we can see

$$
B O=\frac{a}{\cos \theta}
$$

Next, from $\triangle B C D$, the angle $\theta$ is represent by eq. (1) as follows.

$$
\tan \theta=\frac{C D}{B D}=\frac{x-D E}{b}=\frac{x-B F}{b}=\frac{x-(B O-a)}{b}=\frac{x-\left(\frac{a}{\cos \theta}-a\right)}{b}=\frac{\sin \theta}{\cos \theta}
$$

Eq. (2), we can be simplified as follows.

$$
\sqrt{b^{2}+(x+a)^{2}} \sin (\theta+\phi)=-a
$$

Here,

$$
\sin \phi=\frac{-(x+a)}{\sqrt{b^{2}+(x+a)^{2}}}
$$

From eq. (3) and eq. (4), we can obtain following equation.

$$
\theta=\sin ^{-1}\left(\frac{-a}{\sqrt{b^{2}+(x+a)^{2}}}\right)-\phi=\sin ^{-1}\left(\frac{-a}{\sqrt{b^{2}+(x+a)^{2}}}\right)-\sin ^{-1}\left(\frac{-(x+a)}{\sqrt{b^{2}+(x+a)^{2}}}\right)
$$

We can obtain the rebound angle from eq. (5). Here, $a$ is determined from the mirror model size, $b$ is determined by the location of laser displacement sensor.

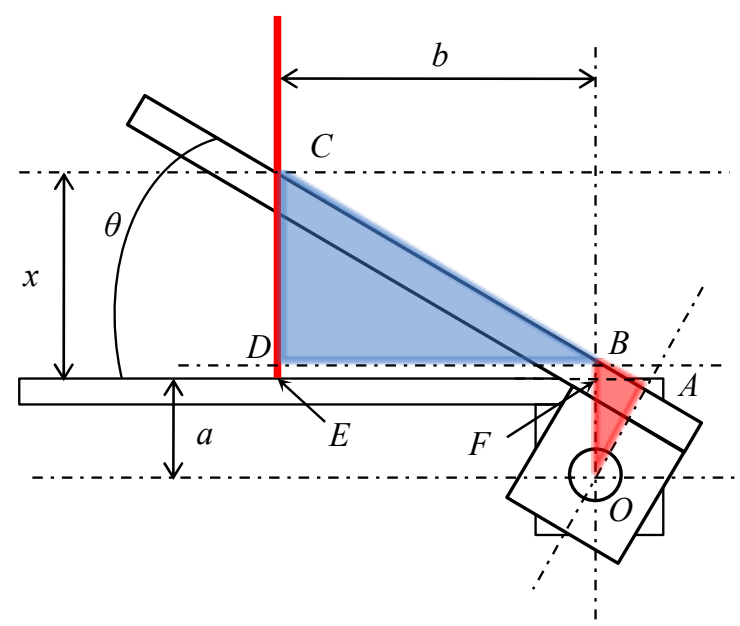

Fig.7 Conversion of displacement into angle

\subsection{Measurement of vibration behavior of the mirror model}

The aim of this measurement is to investigate the mechanism of rebound phenomena. The mirror deformation and vibration of the mirror at the moment of the collision were measured using the same experimental setup shown in Fig.4

Fig.6 shows end elevation of the mirror model. In order to the measurement range of the laser displacement sensor, the mirror model swings down from lower position. The laser displacement sensor was set at 0 deg position when the mirror model contacts the stopper. Thus, the initial position of the mirror model is $18 \mathrm{deg}$. The mirror model freely swings down from initial position, and vertical displacements of the trace of the model's edge were measured. $V_{d}$ is the vertical displacement after the collision with the stopper. The origin of the stopper position is just under the edge of the mirror model as shown in Fig.3. The position of the stopper along y-axis, $y_{s}$ was increased from 0 to $40 \mathrm{~mm}$ by $4 \mathrm{~mm}$ increments, 
and the behavior of the mirror model was measured at different stopper positions to find the smallest and the largest rebound angle. The experiment is performed tree times per each location. It was noted that the differences were very small. Fig.8 shows the measurement positions on the mirror models. For mirror model (a) and mirror model (b), three positions were measured. In the case of mirror model (c) - (f), four points were measured. The measurement positions are noted as No.1 to 4 from the stopper side edge of the mirror as shown in Fig.8.

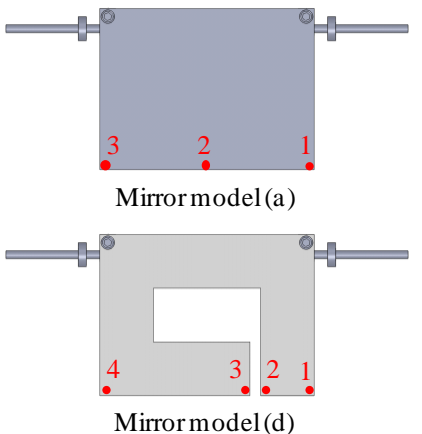

Mirrormodel(d)

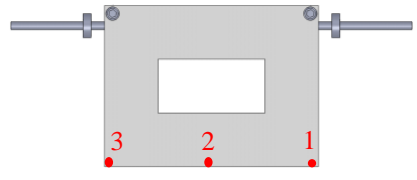

Mirror model(b)

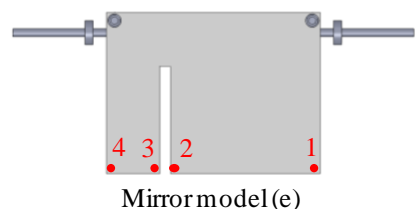

Mirror model(e)

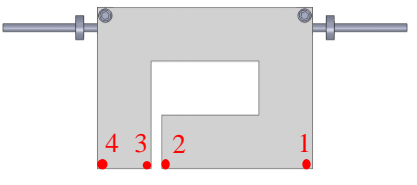

Mirror model(c)

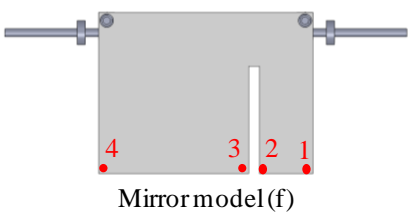

• : Mea surement point

Fig.8 Measurement points

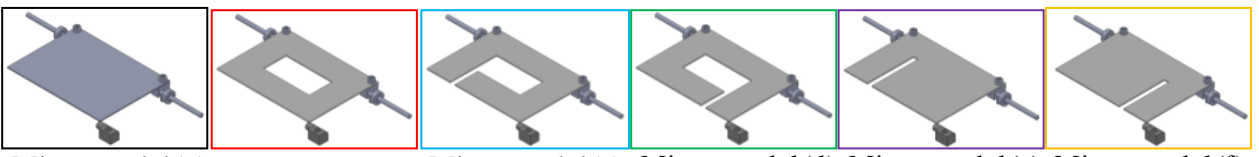

Mirrormodel(a) Mirrormodel(b) Mirrormodel(c) Mirrormodel(d) Mirrormodel(e) Mirrormodel(f)

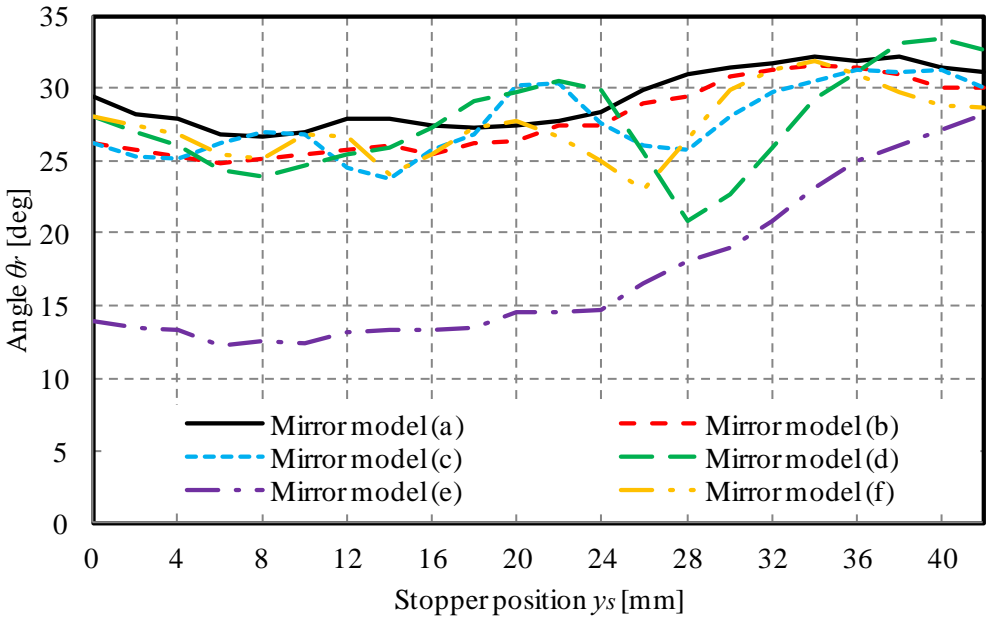

Fig.9 Rebound angle

\section{Experimental results}

\subsection{Rebound angle measurement results}

Fig.9 shows the result of rebound angle and the angle varies as the stopper position changes in all kinds of mirror models.

The maximum rebound angle of the original model, Mirror model (a) is 34 deg at the stopper position $y_{s}=34 \mathrm{~mm}$. On the other hand, the minimum rebound angle of the Mirror model (a) is $27 \mathrm{deg}$ at the stopper position $y_{s}=8 \mathrm{~mm}$. The maximum and the minimum value difference are $6 \mathrm{deg}$. In this case, the rebound angle shows gradual changes against stopper position. 
In the case of Mirror model (b), which has a hole on the center, the maximum rebound angle is $32 \mathrm{deg}$ at $y_{s}=34 \mathrm{~mm}$, and the minimum rebound angle is $25 \mathrm{deg}$ at $y_{s}=6 \mathrm{~mm}$. The maximum and the minimum angle difference is $7 \mathrm{deg}$. These results are similar to the Mirror model (a), (b). Thus, we find that the center hole is lightly effects for the rebound angle.

Next, in the case of Mirror model (c), the maximum rebound angle is about 31 deg at $y_{s}$ $=36 \mathrm{~mm}$ and the minimum rebound angle is $24 \mathrm{deg}$ at $y_{s}=14 \mathrm{~mm}$. The difference is $8 \mathrm{deg}$. In this case, the rebound angle is affected by the stopper position. When $y_{s}$ is large, the rebound angle changes a lot by the stopper position.

In the case of Mirror model (d), the maximum value of rebound angle is $33 \mathrm{deg}$ at $y_{s}$ $=40 \mathrm{~mm}$, and minimum value of rebound angle is $21 \mathrm{deg}$ at $y_{s}=28 \mathrm{~mm}$. Notably, the rebound angle is extremely small at $y_{s}=28 \mathrm{~mm}$, and the Mirror model (d) has the same mass and moment of inertia around the rotational axis as the model (c). But the change of the rebound angle differs from Model (c).

Mirror model (e) has a slit without a rectangle hole. In the case of model (e), the maximum value of rebound angle is $28 \mathrm{deg}$ at $y_{s}=42 \mathrm{~mm}$, and the minimum value of rebound angle is $12 \mathrm{deg}$ at $y_{s}=6 \mathrm{~mm}$. This is the minimum value in all mirror models. Overall, the rebound angle increases with an increase of $y_{s}$. It is consider that the part of left side of mirror model make the model rebound to reduce.

In the case of Mirror model (f), the maximum value of rebound angle is $32 \mathrm{deg}$ at $y_{s}=34 \mathrm{~mm}$, and the minimum value of rebound angle is $23 \mathrm{deg}$ at $y_{s}=26 \mathrm{~mm}$. Overview on the change of rebound angle is similar to model(c) and (d). But it differs widely from model (e), which has the same moment of inertia with model (f).

We can see from the above results that the rebound angle depends on the stopper position. Even if these have same moment of inertia, the rebound angle differs to each other. Therefore, the rebound angle of the mirror model depends on the behavior or the vibration etc. at the moment of collision.

\subsection{Kinetic behavior measurement of the mirror model}

In this section, the kinetic behavior of the edge of the mirror model at the moment of collision at the stopper is discussed. Fig.10 (a) and (b) show measurement results of the Mirror model (a) at the moment of collision correspond to the position $y_{s}=8 \mathrm{~mm}$ and $34 \mathrm{~mm}$ respectively. It is found that the displacement $V_{d}$ of measurement point 1 is zero at the moment of collision, but point 2 and 3 get negative values. Therefore, it is observed that the mirror model deforms elastically by the collision. Also, this results show that the vertical direction deformation at the point 3 is larger than that of point 1 . The time of collision instant has taken as the time when $V_{d} \geq 0$ of all measurement points. After the collision, it is found that the point 1 and 3 vibrate with opposite phase. Vibration amplitude in the case of $y_{s}=8 \mathrm{~mm}$ is larger than that of $y_{s}=34 \mathrm{~mm}$. So that the vibration amplitude is large in the case

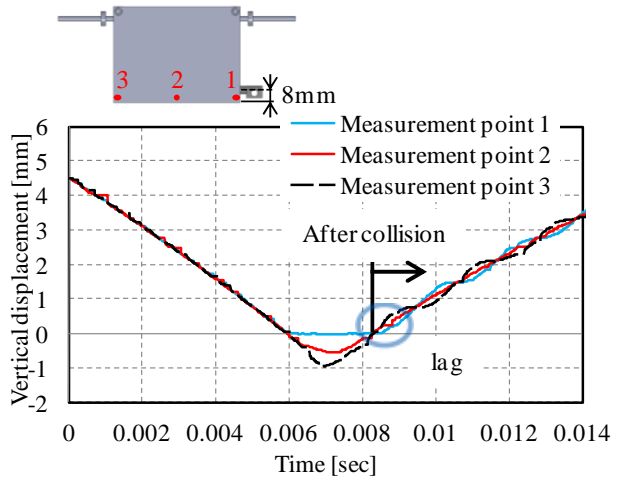

(a) Stopper position $y_{s}=8 \mathrm{~mm}(\theta r=27 \mathrm{deg})$

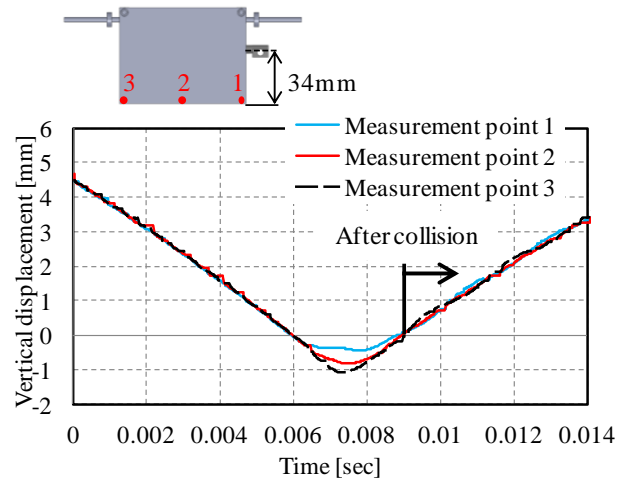

(b) Stopper position $y_{s}=34 \mathrm{~mm}$ ( $\left.\theta r=32 \mathrm{deg}\right)$

Fig.10 Measurement result of the vibration behavior of Mirror model (a) 
of minimum amount of rebound angle. Moreover, we focused our attention on the time of separation from the stopper. It was found that the point 1 separates after separation of other points. As this result, both sides of the mirror begin anti-phase vibration. On the other hand, as shown in Fig.10 (b), the vibration amplitude of both sides of mirror model is very small in the case of maximum rebound angle.

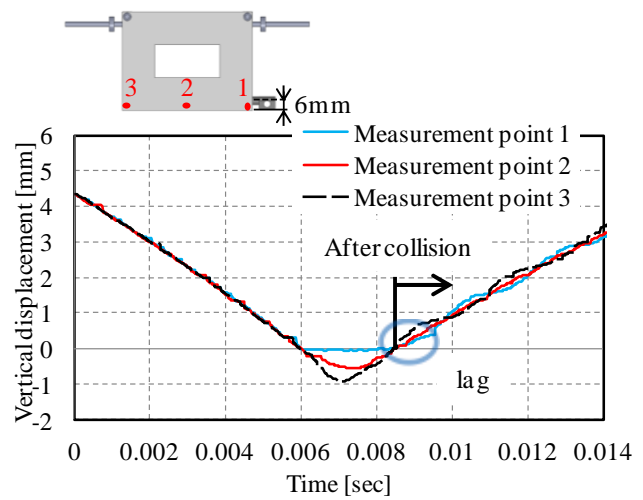

(a) Stopper position $y_{s}=6 \mathrm{~mm}\left(\theta_{r}=25 \mathrm{deg}\right)$

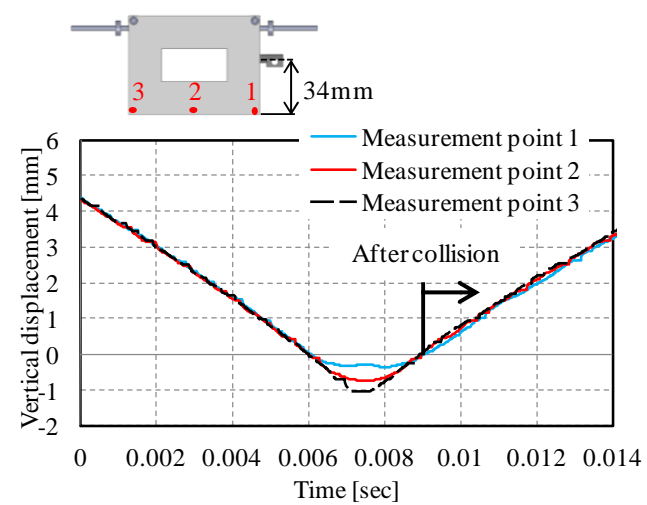

(b) Stopper position $y_{s}=34 \mathrm{~mm}\left(\theta_{r}=32 \mathrm{deg}\right)$

Fig.11 Measurement result of the vibration behavior of Mirror model (b)

Fig.11 (a) and (b) show measurement results of Mirror model (b). Fig.11 (a) and (b) show the rebound behavior in the case of $y_{s}=6 \mathrm{~mm}$ that corresponds to the minimum rebound angle, and $y_{s}=34 \mathrm{~mm}$ that corresponds to maximum rebound angle respectively. The behavior of Mirror model (b) moves approximately same as Mirror model (a). It shows that there are no effects of the center hole in the mirror model on the rebound angle and to the behavior of the model.

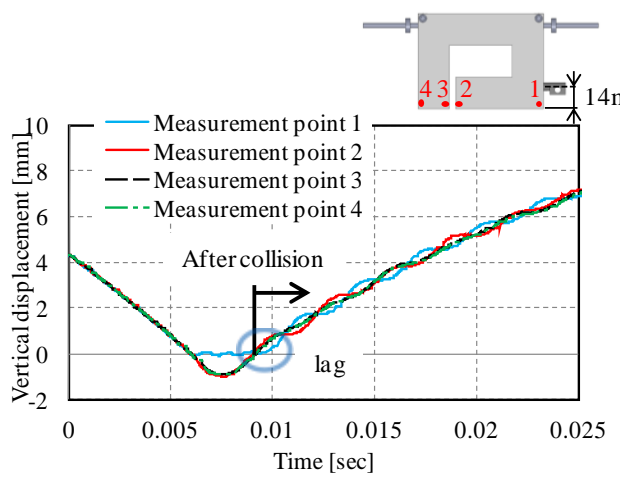

(a) Stopper position: $y_{s}=14 \mathrm{~mm}\left(\theta_{r}=24 \mathrm{deg}\right)$

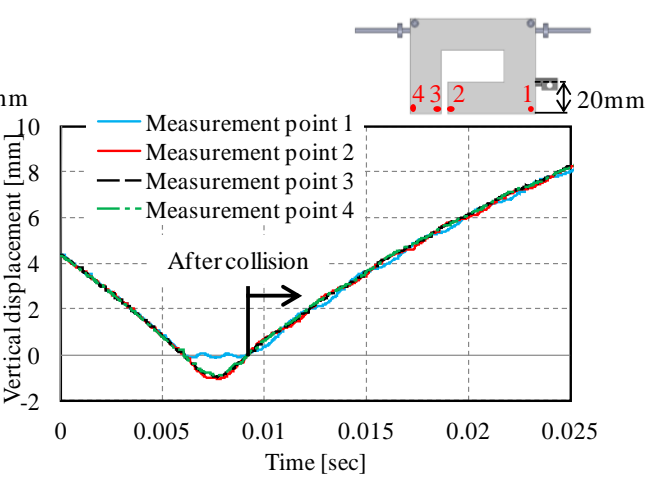

(b) Stopper position: $y_{s}=20 \mathrm{~mm}$ ( $\left.\theta_{r}=30 \mathrm{deg}\right)$

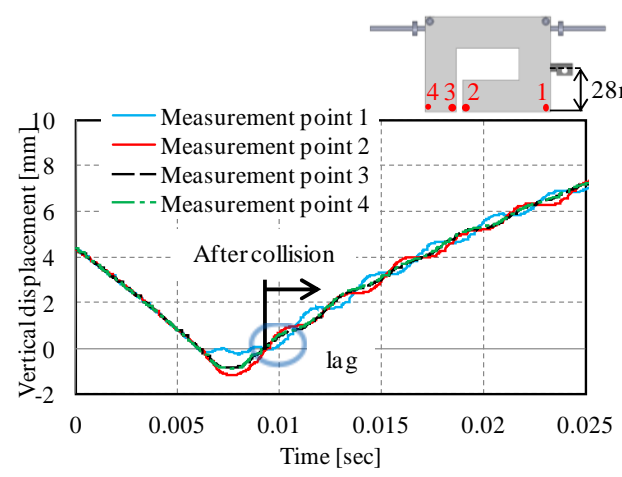

(c) Stopper position: $y_{s}=28 \mathrm{~mm}\left(\theta_{r}=26 \mathrm{deg}\right)$

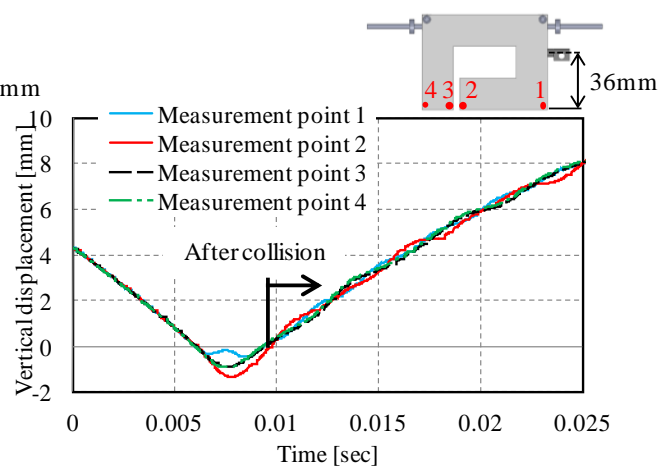

(d) Stopper position: $y_{s}=36 \mathrm{~mm}\left(\theta_{r}=31 \mathrm{deg}\right)$

Fig.12 Measurement result of the vibration behavior of Mirror model (c) 
Fig.12 (a) to (d) show measurement results of the cases of the $y_{s}=14 \mathrm{~mm}, 20 \mathrm{~mm}, 28$ $\mathrm{mm}, 36 \mathrm{~mm}$ of the Mirror model (c) respectively. In the cases of $y_{s}=14 \mathrm{~mm}$ and $28 \mathrm{~mm}$, which has small rebound angle, the edge of mirror models vibrate with large amplitude. These results are similar to the cases of model (a) and (b). Especially, measurement point $2,3,4$ is vibrating with opposite phase to point 1 . Similar with the case of model (a), the point 1 separates after the other points. And also, the point 1 vibrates even when the mirror model is being contacted with the stopper. The vibration characteristics depend on this period of vibration. That is, if the contact time is integral multiple of the vibration period of point 1 , the point 1 moves to the opposite direction against points 2,3,4. Consequentially, both side of the edges vibrate in anti-phase. On the other hand, in the cases of $y_{s}=20 \mathrm{~mm}$ and $36 \mathrm{~mm}$, it is found that the vibration amplitude is relatively small after the collision. In this case, the point 1 and 2,3,4 moves same direction and same phase when it passes $V_{d}=0$.

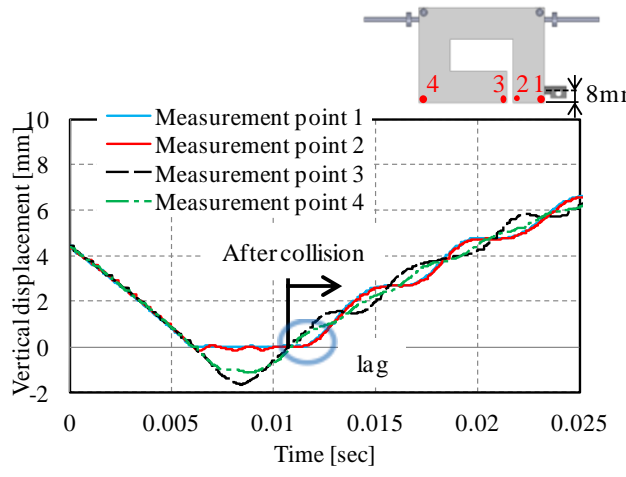

(a) Stopper position: $y_{s}=8 \mathrm{~mm}\left(\theta_{r}=24 \mathrm{deg}\right)$

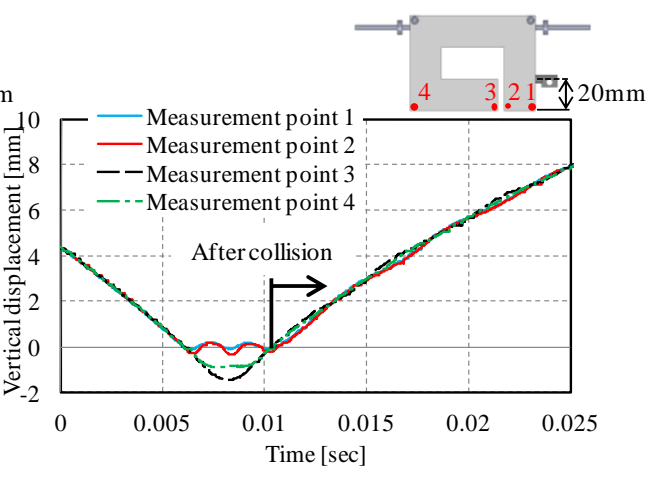

(b) Stopper position: $y_{s}=20 \mathrm{~mm}$ ( $\left.\theta_{r}=30 \mathrm{deg}\right)$

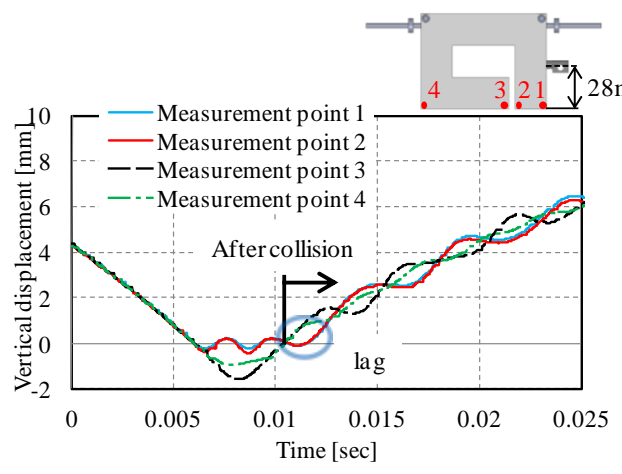

(c) Stopper position: $y_{s}=28 \mathrm{~mm}\left(\theta_{r}=21 \mathrm{deg}\right)$

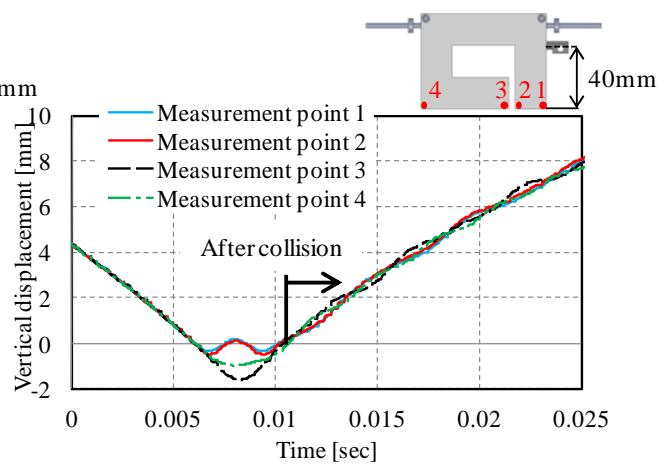

(d) Stopper position: $y_{s}=40 \mathrm{~mm}\left(\theta_{r}=33 \mathrm{deg}\right)$

Fig.13 Measurement result of the vibration behavior of Mirror model (d)

Fig.13 (a) to (d) shows measurement results of the cases of $y_{s}=8,20,28,40 \mathrm{~mm}$ of the Mirror model (d). For the small rebound angle, i.e. $y_{s}=8,28 \mathrm{~mm}$, the edge vibrates with large amplitude. When it passes $V_{d}=0$, it is found that the points 1 and 2 separate after the other points with delay. Compared with Mirror model (c), in the case of Mirror model (d), the points 1 and 2 move approximately the same amount, and it also found that the points 3 and 4 vibrate with larger amplitudes. Thus, it occurs due to larger torsional vibration of this mirror model compared to other models. The torsional vibration makes small the rebound angle of model (d). In cases of $y_{s}=20,40 \mathrm{~mm}$, it finds the vibration does not occur.

Fig.14 (a) and (b) shows the experimental results of the Mirror model (e) at the moment of collision when the stopper is located at $y_{s}=6$ and $42 \mathrm{~mm}$. From both graphs, it can be seen that the points 3 and 4 move approximately the same. The left hand part of mirror model (opposite side of the stopper) vibrates similar to vibration of a cantilever beam. Fig.14 (a) 
shows points 2,3,4 passes $V_{d}=0$ at the same moment. The point 1 does not move at this moment, and then it begin moving to the direction $V_{d}>0$ with a lag. The rebound angle of Mirror model (e) is the smallest compared to other models. But the torsional vibration behavior is similar to the other models despite of having smaller rebound angle. Therefore, it is necessary to consider both kinetic momentum and vibration energy to determine the rebound angle. Fig.14 (b) shows that mirror model does not vibrate when the rebound angle on its maximum value in model (e).

Fig.15 (a) and (b) show experimental results of the Mirror model (f) at the stopper position $y_{s}=26 \mathrm{~mm}, 34 \mathrm{~mm}$. Compared with Fig.15 (a) and (b), it finds that large amplitude of vibration occurs in the case of $y_{s}=26 \mathrm{~mm}$ which has the smallest rebound angle. Fig.15 (a) shows that point 3 and 4 do not vibrate and only move to negative direction and point 1 and 2 vibrates approximately the same. Moreover, points 1 and 2 pass through $V_{d}=0$ after the points 3 and 4. Fig.15 (b) shows that, in the case of maximum rebound angle, the model (e) hardly vibrates after the collision when $y_{s}=34 \mathrm{~mm}$. In this case, the left side part with weak flexural rigidity vibrates optimum timing for reduction rebound angle.

As above, we can conclude that the rebound angle becomes smaller when the mirror model vibrates with large amplitude. On the contrary, the mirror vibration amplitude becomes smaller when the rebound angle is larger. And also, when the rebound angle becomes smaller, we think that excited vibrational mode is torsional mode. The stopper side of the mirror model shows a delay to response after the collision and this delaying phenomenon is directly related to the characteristics of overall vibration of the model. Also, the vibration behavior depends on the mode and the natural frequency of the mirror model.

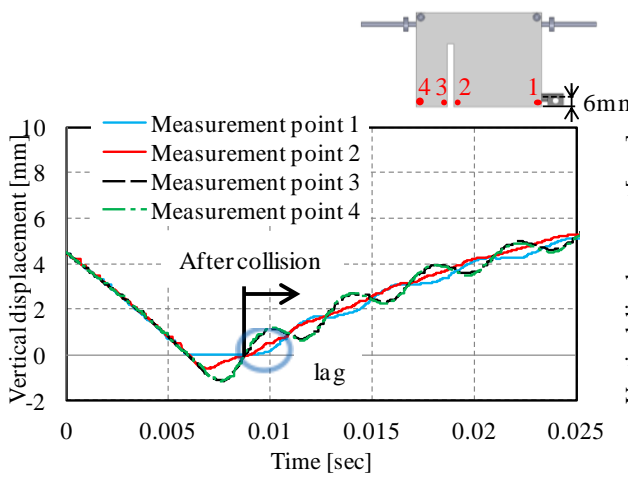

(a) Stopper position: $y_{s}=6 \mathrm{~mm}\left(\theta_{r}=12 \mathrm{deg}\right)$

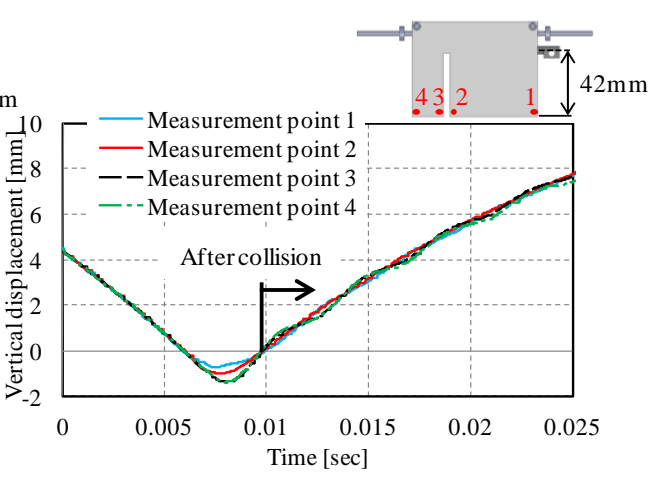

(b) Stopper position: $y_{s}=42 \mathrm{~mm}\left(\theta_{r}=28 \mathrm{deg}\right)$

Fig.14 Measurement result of the vibration behavior of Mirror model (e)

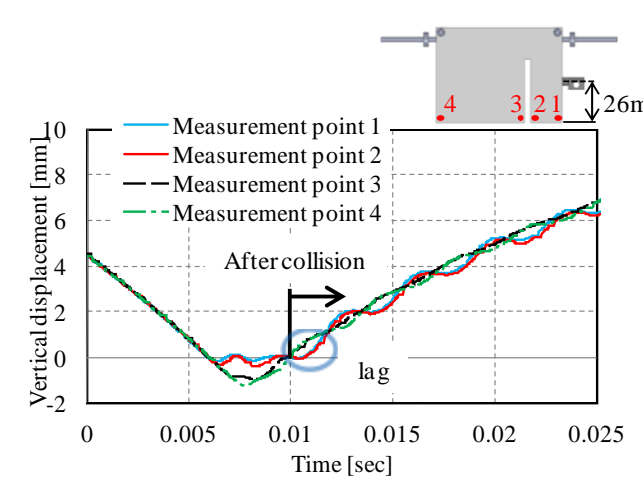

(a) Stopper position: $y_{s}=26 \mathrm{~mm}\left(\theta_{r}=23 \mathrm{deg}\right)$

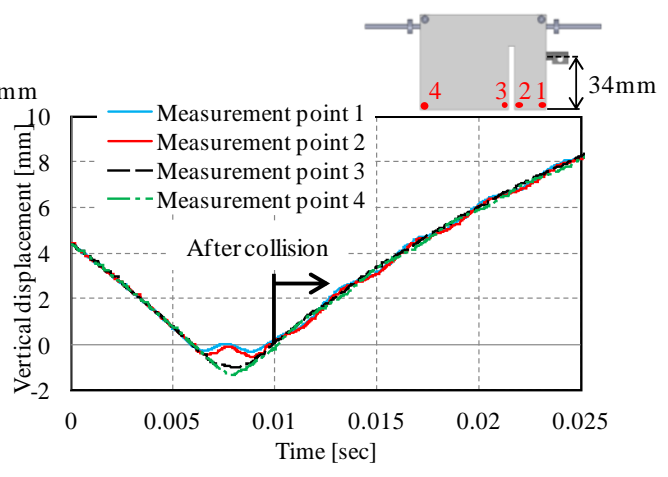

(b) Stopper position: $y_{s}=34 \mathrm{~mm}\left(\theta_{r}=32 \mathrm{deg}\right)$

Fig.15 Measurement result of the vibration behavior of Mirror model (f) 
During the contact time with the stopper, the mirror receives an impact force. Therefore, some natural vibration modes are excited. The above experiment results indicate two or three kinds of vibration mode are occurred on the mirror model in the contact time. We are able to think that the rebound angle is determined by the relationship of these two or three kinds of vibration mode. We are now concluding the forecast method of rebound angle using the modal analysis of the mirror model with the stopper.

\section{Conclusion}

In this research, we investigated collision behavior of the rectangular plate (inner mirror model of SLR camera) with the stopper. We conclude our findings follows,

(1) The rebound angle of the mirror models depends on the stopper position. The stopper position at which the rebound angle represents the minimum value is $8 \mathrm{~mm}$ on the Mirror model (a) (rectangular plate). This result is approximately same in the case of Mirror model (b), which is rectangular plate with rectangular hole in the center. And mirror models with a slit become heavily dependent on the stopper position. The mirror model that has the smallest rebound angle is the mirror model (e), which has a slit on the free side. In the case of the mirror model (e), the rebound angle becomes half value from the case of mirror model (a).

(2) The rebound angle depends on the vibration behavior of the leading edge of mirror model. That is, if there is a phase difference between both sides of leading edges at the moment of leaving the stopper, the mirror model vibrates. We confirmed that the rebound angle become small when the model is in vibrating.

(3) Even with a slit, the vibration amplitude is large when the rebound angle is small. We found that the rebound angle of the models with a slit depends on the behavior of the leading edge at the moment of leaving the stopper.

(4) The rebound angle is varied by stopper position and mirror outline shape. The results indicate possibility of the rebound vibration suppression method at low cost. It is important to design the mirror shape and stopper position. The mirror model shape and the stopper position determine the natural vibration mode. The vibration mode determines the elastic vibration during contact time. The rebound angle is determined by the elastic vibration. Our next purpose is to calculate the rebound angle using the natural vibration mode of the mirror model under contact to the stopper.

\section{References}

(1) Kawazoe, Y., CAE of Tennis Rackets with Impact Phenomena (Prediction of Racket Response and a View of Restitution in Racket-Ball Impact) (in Japanese), Transactions of the Japan Society of Mechanical Engineers, Series C, Vol.58, No.552, (1992), pp.143-150.

(2) Kawazoe, Y., Analysis of Coefficient of Restitution during a Nonlinear Impact between a Ball and Strings Considering Vibration Modes of Racket Frame (in Japanese), Transactions of the Japan Society of Mechanical Engineers, Series C, Vol.59, No.562, (1993), pp.76-83.

(3) Yamaguchi, T., Yamamoto, T., Tsunoda, M., Iwatsubo, T., A Study for Optimum Design of Tennis Racket (in Japanese), Transactions of the Japan Society of Mechanical Engineers, Series C, Vol.61, No.588, (1995), pp. 197-202

(4) Kawazoe, Y., Tomosue, R., Prediction of a Sweet Area on a Racket Face in a Tennis Impact 
(Restitution Coefficient, Rebound Power Coefficient and Ball Post-Impact Velocity) (in Japanese), Transactions of the Japan Society of Mechanical Engineers, Series C, Vol.64, No.623, (1998), pp. 72-78

(5) Kawazoe, Y., Effect of Racket Frame In-Plane Hoop Vibrations on the Coefficient of Restitution and Energy Losses in Tennis Rackets (in Japanese), JSME Symposium on Sports Engineering: Symposium on Human Dynamics 2006, No.06-35, (2006), pp.130-135.

(6) Hata, H., Utsuno, H., Matsuhisa, H., Modal analysis of a metal baseball bat considering the restitution of ball (in Japanese), JSME Symposium on Sports Engineering: Symposium on Human Dynamics 2004, No.04-26, (2004), pp.89-94

(7) Matsumoto, H., Sasaki, T., Saitoh, K., Rebound Vibration of a Rotary Supported Plate Hit a Stopper (in Japanese), Dynamics \& Design Conference 2011, (2011), No.424 